\title{
NERNST EFFECT IN TI-Ba-Ca-Cu-O SUPERCONDUCTING FILMS DUE TO INFRARED LASER HEATING
}

\author{
H. Lengfellner, A. Schnellbögl, J. Betz, K. F. Renk, \\ and W. Prettl \\ Institut für Angewandte Physik \\ Universität Regensburg \\ D-8400 Regensburg, West Germany
}

Received April 13, 1990

\begin{abstract}
A Nernst effect has been observed in a high temperature superconductor for the first time. Irradiating superconducting $\mathrm{Tl}-\mathrm{Ba}-\mathrm{Ca}-\mathrm{Cu}-$ $\mathrm{O}$ thin films by short pulses of a TEA-CO $\mathrm{CO}_{2}$ laser, a photovoltaic signal is detected perpendicular to a magnetic field applied parallel to the film surface. The signal is attributed to magnetic flux line depinning and flux line transport driven by the laser induced temperature gradient. The results are described by thermal flux line activation leading to a calculated distribution of pinning energies from $100 \mathrm{~K}$ to $4000 \mathrm{~K}$.
\end{abstract}

Key words: Nernst effect, high temperature superconductor, flux pinning, pinning energies.

\section{Introduction}

One of the promising applications of high temperature superconductors is as broadband optical detectors particularly suitable for the infrared spectral range. In previous investigations bolometric signals following the derivative of the resistance-temperature relation have been observed [1]. In granular films, a high-speed non-thermal response has been detected [2]. The latter effect was attributed to an optically induced destruction of the wave function phase coherence in a random network of Josephson junctions [3]. These measurements were performed in a photoconductive mode of operation: the samples were biased by a current source and the optically induced voltage change across the superconductor was measured. In the present 
work we report on a new class of optical effects giving rise to a photovoltaic signal in response to a radiation pulse .

Superconducting Tl-Ba-Ca-Cu-O thin films were irradiated by pulses of a TEA-CO ${ }_{2}$ laser generating a temperature gradient perpendicular to the film surface. Without any bias current, a voltage signal was observed perpendicular to both the temperature gradient and a small magnetic field applied parallel to the film surface. This photovoltaic signal is attributed to a Nernst effect due to magnetic flux depinning and flux transport driven by the laser induced temperature gradient. The magnetic field creates the transverse voltage in a manner analogous to the Hall effect. This Nernst effect may offer a novel method to investigate magnetic flux tube motion in high temperature superconductors as well as demonstrating a new principle of infrared detection. The dynamics of flux tubes is an issue of current importance $[4,5]$.

\section{Experimental Technique}

The measurements were carried out on polycrystalline Tl-Ba$\mathrm{Ca}-\mathrm{Cu}-\mathrm{O}$ superconducting films of about $1 \mu \mathrm{m}$ thickness showing transition temperatures near $100 \mathrm{~K}$. The film preparation procedures and details on the morphology of the films were described elsewhere $[6,7]$. The films were deposited on $10 \times 10 \times 1 \mathrm{~mm}^{3} \mathrm{SrTiO}_{3}$ substrates and had bridge-like patterns. The experimental arrangement is shown in Fig. 1. The magnetic field was generated by a stack of small CoSm solid state magnets yielding a field strength of about $0.1 \mathrm{~T}$ with the field vector parallel to the plane of the film. Contacts

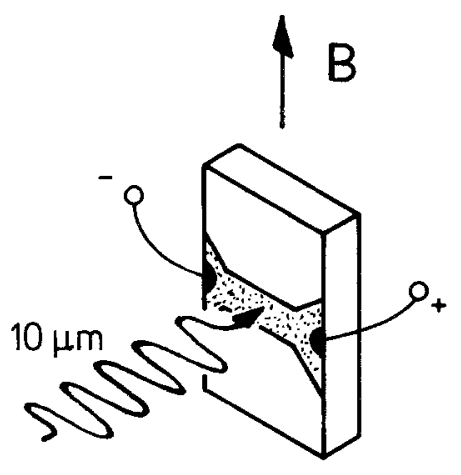

Fig. 1 - Experimental arrangement. The contact areas were protected against irradiation by metallic shields. 
for measuring the transverse voltage were made with silver epoxy paint. The contact regions were shielded by a metallic mask to prevent irradiation of the contacts. The whole arrangement was placed in a temperature variable cryostat with optical access. The films were irradiated with TEA-CO 2 laser pulses (duration $100 \mathrm{~ns}$, $1 \mathrm{~s}^{-1}$ repetition rate, $\lambda \approx 10 \mu \mathrm{m}$ ). The energy of the laser pulses was varied by calibrated attenuators, the maximum pulse energy density at the film surface was about $20 \mathrm{~mJ} / \mathrm{cm}^{2}$. Signals were recorded with a fast storage oscilloscope.

\section{Experimental Results}

In Fig. 2 two signal pulses are shown for opposite directions of the applied magnetic field demonstrating that upon reversing the orientation of the field the polarity of the signal voltage changes as expected for a Nernst effect [8].

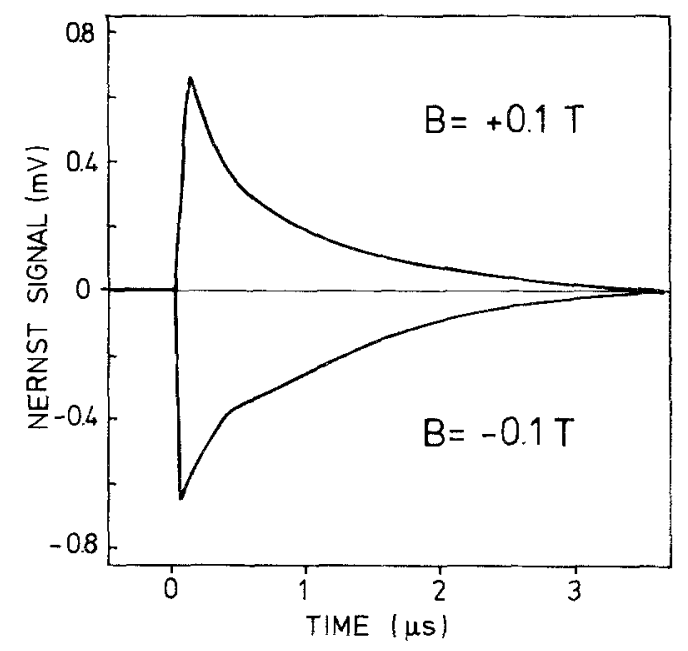

Fig. 2 - Two signal pulses for the magnetic field pointing in opposite directions in the plane of the the film. $B>0$ corresponds to the orientation of $B$ with respect to the signal polarity shown in Fig. 1. Laser pulse energy density $\mathrm{E}_{P}=$ $8 \mathrm{~mJ} / \mathrm{cm}^{2}$, initial film temperature $T_{I}=30 \mathrm{~K}$. 


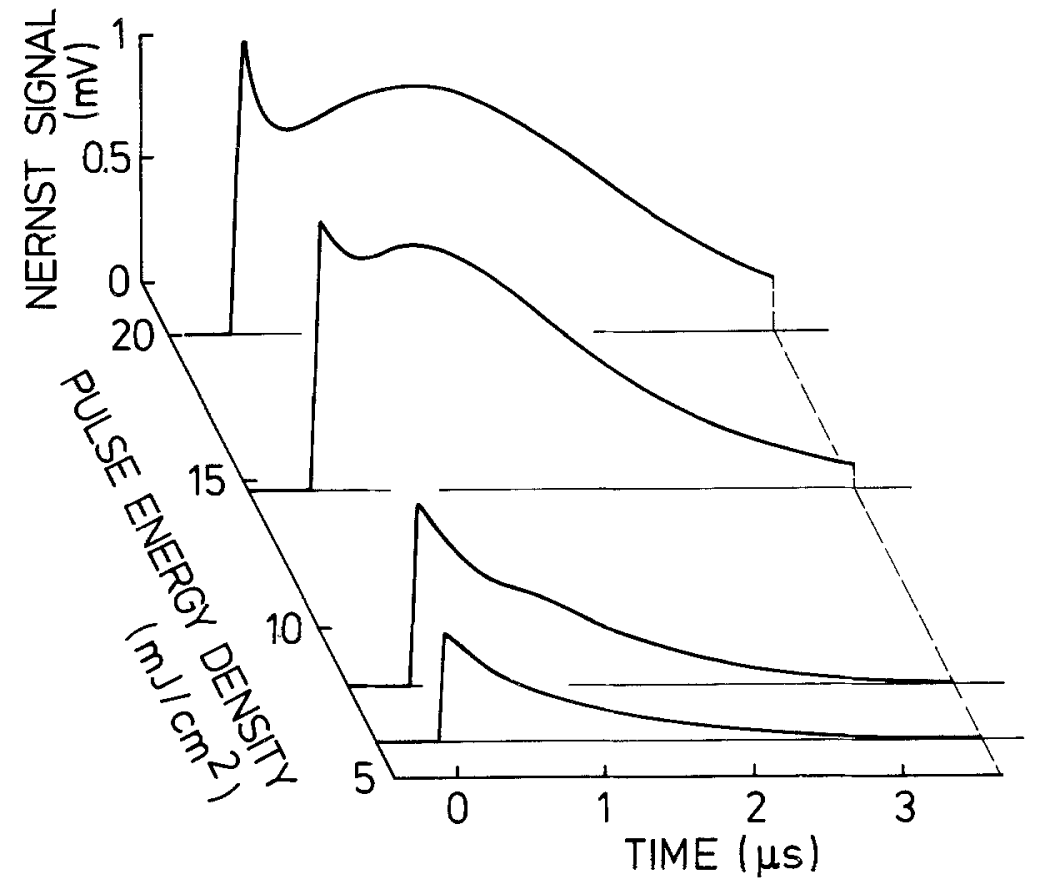

Fig. 3 - Nernst signal pulses for various laser pulse energy densities, initial film temperature $\mathrm{T}_{I}=30 \mathrm{~K}$.

In Fig. 3 a series of recordings for different laser pulse energy densities and an initial temperature of the sample of $T_{I}=30 \mathrm{~K}$ is plotted. The time resolved Nernst signals with maximum voltages up to $1 \mathrm{mV}$, show the same fast rise as the laser pulse and a slow decrease time of about $1 \mu s$. As the Nernst voltage is proportional to the temperature gradient $\nabla T$ [9], the signals indicate the dynamics of the build-up and decay of a temperature gradient accross the film. The temperature gradient assumes a maximum at the end of the laser pulse and decays afterwards. With increasing pulse energy part of the film even reaches a final temperature $T>T_{c}$. In this case we observe, after a first maximum of the Nernst signal, a decrease of the signal due to the film becoming partially normal conducting. The Nernst signal increases again at larger times when the film has cooled down so that $T<T_{c}$ everywhere. With further increasing pulse energy, the second peak shifts to a longer time indicating an increase of cool-down time, whereas the signal height stays approximately constant. 


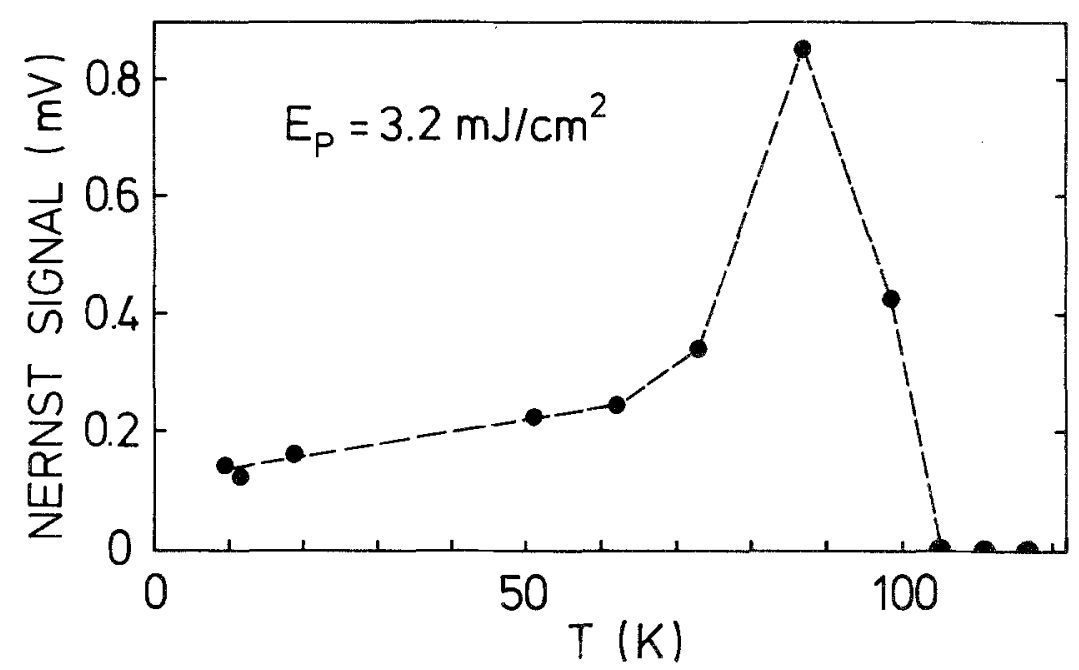

Fig. 4 - Signal height for low laser pulse energy density of $E_{P}=$ $3.2 \mathrm{~mJ} / \mathrm{cm}^{2}$ as function of temperature $T_{I}$; the signal vanishes if the sample becomes normal conducting.

In Fig. 4 the signal height measured at a constant nonsaturating laser pulse energy of $3.2 \mathrm{~mJ} / \mathrm{cm}^{2}$ as a function of $T_{I}$ is shown. The signal height is nearly constant at low temperatures, shows a maximum near $T_{c}$ and vanishes above $T_{c}$. The height of the Nernst voltage versus laser pulse energy density $E_{P}$ is plotted in Fig. 5 for various initial sample temperatures $T_{I}$. The signals show a superlinear onset at low energies, increase with a slope depending on $T_{I}$ and finally saturate at about the same maximum value for all $T_{I}$ 's. The saturation of the signal indicates that for high pulse energies, $T_{c}$ of the superconducting film is always exceeded.

\section{Discussion}

The Nernst voltage is generated by flux motion between two contact points (10). The voltage is proportional to the rate at which flux crosses a curve joining the contacts. The rate depends on the fluxon velocity which is proportional to the thermal force $f_{t h}=-S \nabla T(9)$ where $S$ is the fluxon transport entropy and $\nabla T$ is the temperature gradient, and on the depinning probability, i.e. the thermal activated hopping rate $\nu=\nu_{o} \exp \left(-T_{P} / T_{F}\right)$ of fluxons. In this expression $\nu_{o}$ is the attempt frequency, $T_{P}$ the pinning energy 


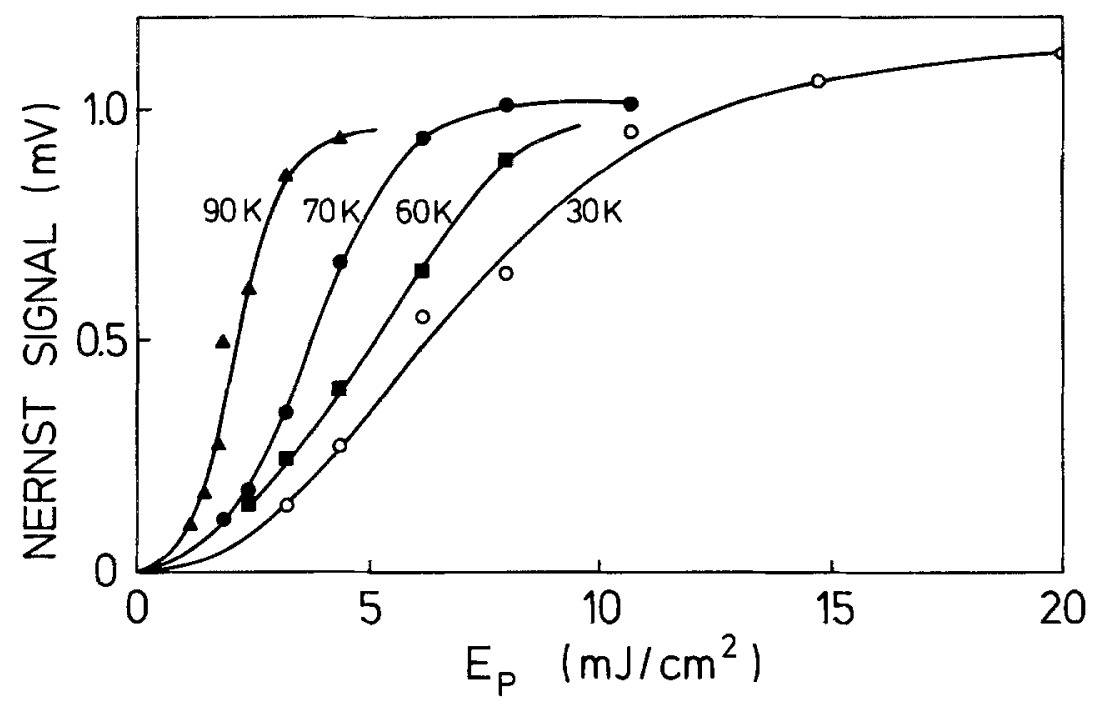

Fig. 5 - Signal height vs irradiation energy density $E_{P}$, for various initial film temperatures $T_{I}$.

and $T_{F}$ the film temperature.

Because the fluxons move from the hot (front) film surface to the colder film reverse and because this motion depends upon flux depinning in this colder region of the film, we chose for $T_{F}$ a film temperature near the interface between film and substrate. For small laser pulse energies, as long as specific heat and diffusivity are constant, the film temperature rise is proportional to the laser pulse energy. From a heat transfer model (11), a measurement of the diffusivity of our film (12) and diffusivity values of $\mathrm{Y}-\mathrm{Ba}-\mathrm{Cu}-\mathrm{O}$ films (13), we obtain for our film $\Delta T=\nabla T \cdot d=A\left(T_{I}\right) \cdot E_{P}$ and $T_{F}=B\left(T_{I}\right) \cdot E_{P}+T_{I}$ where $\Delta T$ is the temperature difference between film surfaces, $d$ the film thickness, $E_{P}$ the energy density of the laser pulse, $T_{I}$ the initial film temperature, and $A=1.9 \times 10^{3} \mathrm{Kcm}^{2} / \mathrm{J}$, $B=3.5 \times 10^{3} \mathrm{Kcm}^{2} / \mathrm{J}$ at $T_{I}=60 \mathrm{~K}$, for example. These values change little for higher $T_{I}$ values; for low temperatures the effect of the strongly changing specific heat must be considered. We will describe our results using an expression

$$
\begin{aligned}
U_{N} & =C \cdot \nabla T \cdot S \cdot \nu_{o} \exp \left[-T_{P} / T_{F}\right] \\
& =C^{\prime} \cdot E_{P} \cdot \exp \left[-T_{P} \cdot\left(B \cdot E_{P}+T_{I}\right)^{-1}\right]
\end{aligned}
$$

for the Nernst signal voltage $U_{N}$ with proportionality constants $C, C^{\prime}$, and with the relations given above for $\nabla T$ and $T_{F}$ for small 


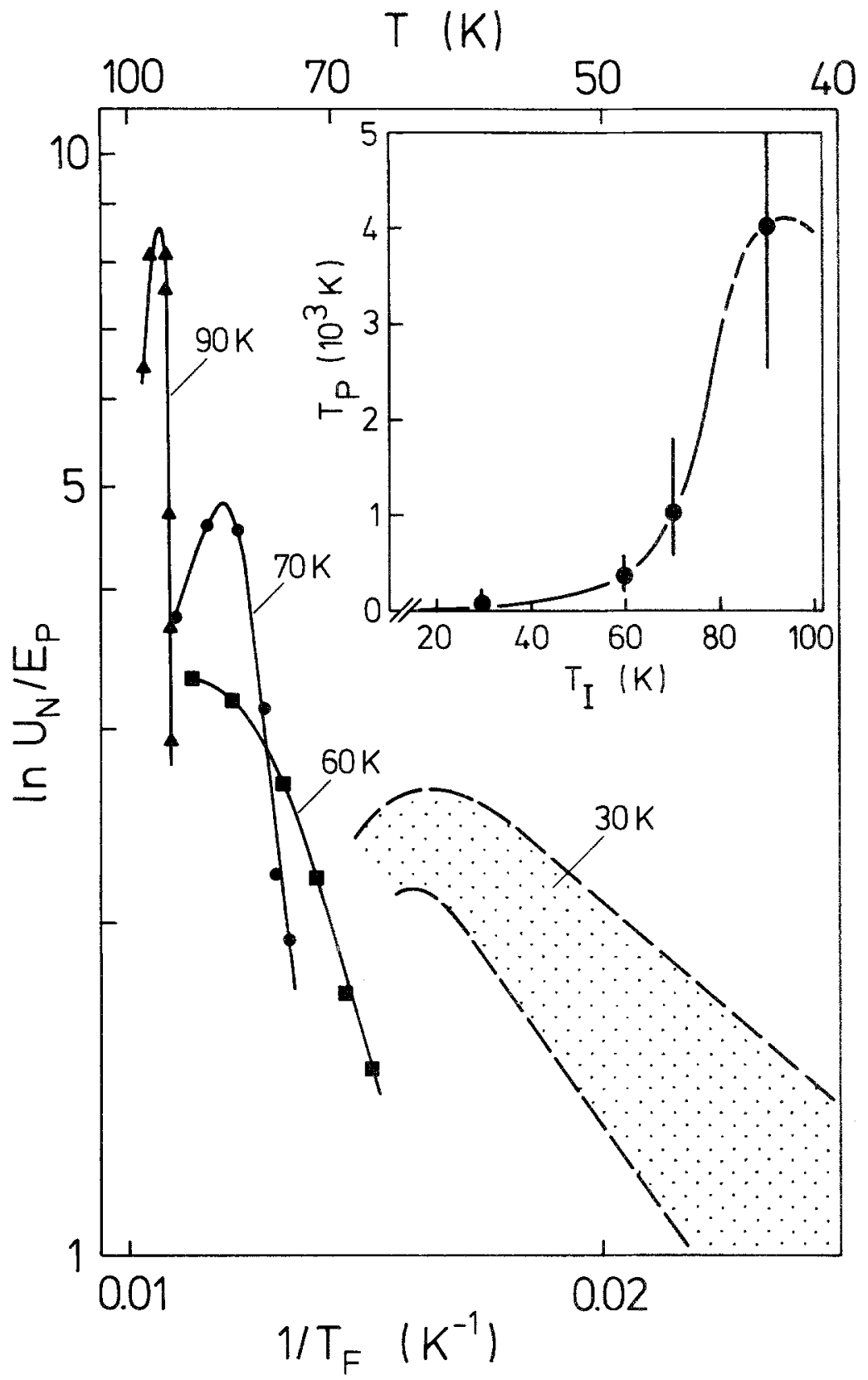

Fig. 6 - Dependence of the normalized Nernst signal $U_{N} / E_{P}$ on film temperature $T_{F}$, for various initial film temperatures $T_{I}$. The inset shows values of pinning energy $T_{P}$ obtained at different $T_{I}$. 
$E_{P} . S$ is assumed to be constant.

A logarithmic plot of $U_{N} / E_{P}$ against $1 / T_{F}$ for various values of $T_{I}$ is shown in Fig. 6. At small laser energies the curves show constant slopes from which we deduce values for $T_{P}$. With increasing laser energy, $T_{F}$ increases and the curves bend due to saturation of the signal. Due to uncertain $T_{F}$ for the $T_{I}=30 \mathrm{~K}$ measurement, we give an estimated region for the slope of this curve (Fig. 6). The inset shows values of $T_{P}$ for the different $T_{I}$. Our results indicate a distribution of pinning centers with different pinning energies ranging from low values ( $\leqslant 100 \mathrm{~K}$ ) up to values of about $4000 \mathrm{~K}$. At low temperatures only loosely bound fluxons are depinned and contribute to the signal. At higher temperatures strongly bound flux is also thermally activated leading to a strong increase of the Nernst signal.

\section{Conclusion}

In conclusion, we have observed, for the first time, a Nernst effect in a high temperature superconductor by applying pulsed laser heating of thin films. An analysis of the Nernst signals based on flux motion driven by thermal forces and thermally activated hopping delivers values of the pinning energy $T_{P}$. We find, for a Tl-Ba$\mathrm{Ca}-\mathrm{Cu}-\mathrm{O}$ thin film, a distribution of pinning energies ranging from $\lesssim 100 \mathrm{~K}$ to several thousand $\mathrm{K}$.

\section{Acknowledgements}

The work was supported by the EC and by the Bundesministerium für Forschung und Technologie.

\section{References}

[1] M.G. Forrester, M. Gottlieb, J.R. Gavaler, and A.I. Braginski, Appl. Phys. Lett. 53, 1332 (1988).

[2] A. Frenkel, M.A. Saif, T. Venkatesan, Chinlon Lin, X.D. Wu, and A. Inam, Appl. Phys. Lett. 54, 1594 (1989); J.C. Culbertson, U. Strom, S.A. Wolf, P. Skeath, E.J. West, and W.K. Burns, Phys. Rev. B 39, 12359 (1989).

[3] M. Leung, U. Strom, J.C. Culbertson, J.H. Claassen, S.A. Wolf, and R.W. Simon, Appl. Phys. Lett. 50, 1691 (1987).

[4] A. Gupta, P. Esquinazi, H.F. Braun, and H.W. Neumüller, Phys. Rev. Lett. 63, 1869 (1989).

[ 5] T.T.M. Palstra, B. Batlogg, L.F. Schneemeyer, and J.V. Waszczak, Phys. Rev. Lett. 61, 1662 (1989). 
[6] J.Betz, H. Lengfellner, E. Duschl, K. Meidenbauer, and K.F. Renk, Physica C 162 - 164, 133 (1989).

[ 7] H. Lengfellner, J. Betz, and K.F. Renk, Appl.Phys. A48, 501 (1989).

[8] H.B. Callen, Thermodynamics, Wiley 1960.

[9] R.P. Huebener, Magnetic Flux Structures in Superconductors, Springer Series in Solid-State Sciences 6, eds. M. Cardona, P. Fulde, and H.-J. Queisser, Berlin (1979).

[10] B.D. Josephson, Physics Letters 16 (1965) 242.

[11] H.S. Carlslaw and J.C. Jaeger, Conduction of Heat in Solids, Clarendon Press, Oxford (1959).

[12] A. Schnellbögl, H. Lengfellner, and W. Prettl, to be published.

[13] L. Gomes, M.M.F. Vieira, S.L. Baldochi, N.B. Lima, M.A. Novak, N.D. Vieira Jr., and S.P. Morato, J. Appl. Phys. 63 (1988) 5044. 